\title{
ENSEÑANZA DEL DERECHO Y PROFESIONES JURÍDICAS
}

\author{
Josep M. Vilajosana* \\ Universidad Pompeu Fabra
}

\begin{abstract}
- a enseñanza del derecho parece que alguna relación debería mantener con la forma de entender las profesiones jurídicas. Esta indudable relación es la que me permitirá conectar en este breve escrito algunos de los análisis de Francisco LAPORTA y de Liborio HIERRO. Del primero tomaré sus textos sobre la enseñanza del derecho y del segundo los que dedica a las profesiones jurídicas y, en especial, a la enseñanza de la deontología jurídica.
\end{abstract}

\section{ENSEÑANZA, MODELO DE JURISTA Y BOLONIA}

A continuación centraré mi atención en dos de las aportaciones de LAPORTA: la Introducción a un libro sobre la enseñanza del derecho, editado por el autor ${ }^{1}$, y un texto que plantea de manera crítica el proceso de Bolonia, en general, y el de su aplicación a la enseñanza jurídica, en particular ${ }^{2}$.

La pretensión principal del primer texto es, en palabras del autor, «formular con un poco más de precisión los términos del debate» (p. 14). En mi opinión consigue el objetivo sobradamente y en muy pocas páginas. Quisiera simplemente destacar dos observaciones importantes:

1. Hay tres modelos de universidad: investigadora, profesional y educadora. El énfasis en uno u otro de estos modelos ideales, dará pie a un tipo de Facultad de derecho distinto. LAPORTA sostiene que el predominio del modelo investigador daría lugar a unos estudios socialmente poco útiles y cuyo modelo de jurista vendría a ser el profesor de derecho (o el cultivador de las ciencias jurídicas). El modelo profesional tendría en el punto de mira el ejercicio de las profesiones jurídicas y al jurista se le enseñaría «no a hacer ciencia, sino a aplicar los conocimientos científicos» (p. 17). Por último, la universidad educadora, planteada como el escalón superior de la formación que ha venido recibiendo el alumnado en etapas anteriores, daría cabida

* Catedrático de Filosofía del Derecho en la Universidad Pompeu Fabra: josep.vilajosana@upf.edu.

1 F. LAPORTA, «A modo de introducción: la naturaleza de las reflexiones sobre la enseñanza del derecho, en F. LAPORTA (ed.), La Enseñanza del Derecho, Anuario de la Facultad de Derecho de la Universidad Autónoma de Madrid, 6, 2003, 13-24.

2 F. LAPORTA, «El proceso de Bolonia y nuestras Facultades de Derecho», Revista de Estudios jurídicos, núm. 10, 2010, 1-10. 
a materias no específicamente profesionalizadoras ni de dogmática jurídica, como historia, sociología, economía, filosofía, etc. Según LAPORTA, el predominio de este modelo significaría en última instancia poner en entredicho el postulado de la autonomía del derecho «como objeto de conocimiento y técnica específica de control social» (p. 18).

2. Para enseñar el «derecho» tiene que tenerse claro un concepto de derecho. Según este autor, aunque se reconoce mayoritariamente el peso de los principios jurídicos en el razonamiento de los juristas y la relevancia que estos han adquirido a través de la positivización en las constituciones de nuestro entorno, «seguimos enseñando el derecho igual que antes, y supongo que esperando que por una suerte de ciencia infusa los jueces y magistrados sepan realizar esas nuevas tareas» (p. 19). Estas «nuevas tareas» podrían ser, eventualmente, las que se centrarían en la argumentación jurídica, que conllevaría no solo el manejo de los tradicionales criterios interpretativos, sino toda la problemática que supone reconocer que los principios jurídicos contienen pautas morales.

Pero quien podría asumir tales cambios (y otros que aquí no he mencionado) y ser su impulsor —el profesorado en formación- es precisamente el que está pagando más caro la apuesta poco decidida de las autoridades por este nuevo modelo. En realidad, el nuevo modelo que se ha propuesto desde la autoridad competente se ha concretado en el llamado proceso de Bolonia, que es el objeto de crítica del segundo texto de LAPORTA que voy a comentar a continuación.

La crítica contundente a la que somete LAPORTA al proceso de Bolonia queda muy bien reflejada en la metáfora que emplea. La aplicación práctica de este proceso ha sido como una especie de trampantojo, pura fachada colorista, que en realidad esconde que tras la pared no hay nada. Denuncia que la convergencia con los demás países europeos propiciada por Bolonia es puramente formal y nada de lo que en este proceso se recoge garantiza que se alcancen los niveles de las mejores universidades. ¿Qué se podría hacer al respecto? Aquí LAPORTA sostiene que hay cuatro problemas que habría que afrontar para conseguir esta meta. Son los que llama «gatos sin cascabel» porque nadie se atreve a ponérselo:

Primero, la limitación o selección seria del acceso de los estudiantes a los estudios universitarios [...]. El resultado es que los peores estudiantes que tenemos son precisamente los de derecho. Eso lo saben, y lo dicen claramente, todos los colegas. [...]. Segundo «gato» sin cascabel, consecuencia del anterior: el excesivo número de estudiantes por curso en ciertos estudios (muy claramente en los de derecho). Nuestros estudiantes no solo no están entre los mejores, sino que son demasiados. [...]. El tercer problema también se relaciona con ello: la cuestión de la financiación de la Universidad y lo escuálido de las tasas que pagan los estudiantes (en torno al 15\% del coste real de su plaza). Esto determina un disparate mayúsculo: que sea el número y no la calidad de los estudiantes lo que determina la financiación de la universidad. Pues bien, es preciso decirlo con toda claridad: es esa y no otras la causa de que nuestras universidades no aparezcan en ninguna lista seria. Y por último, está la selección y valoración —científica y también económica- de los profesores y la promoción de una cultura de la seriedad y el rigor en la carrera docente. Temo que a este respecto vamos precisamente en la dirección contraria. (pp. 5 y 6). 


\section{PARA SEGUIR DEBATIENDO}

El entero planteamiento de LAPORTA es muy sugerente. Coincido en su mayor parte tanto con la descripción como con alguna de sus propuestas. Sin embargo, con la finalidad de continuar con el debate, me gustaría poner de relieve una posible tensión de su planteamiento y algunos puntos más concretos de discrepancia.

Por lo que hace a la tensión, una cosa es afirmar que el proceso de Bolonia y los objetivos que persigue están en mayor o menor medida equivocados, y otra muy distinta sostener que si se dieran las cuatro condiciones citadas (los «cascabeles») se conseguiría la meta perseguida. Es decir, o lo que Bolonia pretende alcanzar es inadecuado (lo cual parece constituir un punto central de la crítica de LAPORTA), en cuyo caso no hay condición que transforme tales objetivos en correctos, o bien estos son deseables, aunque no pueden alcanzarse a menos que se cumplan las mencionadas condiciones.

Por otro lado, debo decir que mi experiencia con el proceso boloñés ha sido más positiva de lo que se sugiere en el texto comentado e incluso de lo que yo mismo pensé en un principio ${ }^{3}$. Es cierto que esa experiencia ha tenido lugar en una Facultad en la que con el tiempo se ha incrementado la nota de corte y, por tanto, los estudiantes que ingresan en derecho son de un nivel bastante alto en términos relativos. Desde mi punto de vista este es el punto clave para conseguir que Bolonia (en realidad cualquier sistema) funcione adecuadamente. No sé si en todas partes se pueden dar las circunstancias requeridas para esa criba (que en realidad en nuestro caso solo obedece al juego de la oferta y la demanda), aunque tal vez haya que reconocer que es difícil tener cincuenta buenas facultades de derecho (ni de cualquier otro tipo de estudios).

Algo que sí comparto totalmente con LAPORTA es la preocupación por la formación de los jóvenes profesores, pero mi perspectiva es algo distinta. Creo que hay, hoy por hoy, una cantidad creciente de profesores en formación de un nivel muy alto, que han completado su aprendizaje en las mejores universidades del mundo. Lo que no existe, en mi opinión, es una voluntad clara (ni de los poderes públicos, ni de las propias universidades) de incentivar convenientemente a este profesorado. Esta carencia supone, entre otras cosas, arrojar por la borda cantidades ingentes de recursos públicos. Es muy frustrante para alguien que se ha formado del mejor modo posible (lo cual consume tiempo, esfuerzo y dedicación casi exclusiva al estudio), ver que después de todo el sistema universitario lo expulsa de la vida académica o lo mantiene en precario de un modo totalmente injustificado.

\section{PROFESIONES JURÍDICAS Y ENSEÑANZA DE LA DEONTOLOGÍA}

Como decía al inicio, la enseñanza del derecho no se puede desvincular de la idea que se tenga de las profesiones jurídicas. Ello es así en mayor medida si se adopta un modelo profesionalizador de enseñanza del derecho, del que hablaba LAPORTA. Pero cuál sea este dependerá obviamente de cómo se defina a la profesión jurídica.

3 Me refiero a mi experiencia como profesor, pero también como decano de la Facultad de Derecho de la Universidad Pompeu Fabra durante seis años (2010-2016). 
HierRo ha ofrecido una definición de este tipo ${ }^{4}$. Así, propone entender como profesiones jurídicas «aquellas actividades cuya función consiste en la elaboración técnica de normas jurídicas, su aplicación y la prevención y resolución de conflictos jurídicos» (p. 35). Por ello, «lo característico de las profesiones jurídicas sería la elaboración y aplicación de normas jurídicas. El jurista (como sinónimo de «profesional jurídico») elabora el elemento técnico de las normas aunque, con la mayor frecuencia, no es la autoridad que las aprueba» (p. 36). Después comentaré algo al respecto, pero ahora concentraré mi atención en el otro aspecto que quería resaltar de los trabajos de HIERRO. Se trata del asunto más específico de la enseñanza de la deontología profesional ${ }^{5}$.

Como bien dice HiERRO, la enseñanza de la deontología profesional se ha limitado hasta ahora a ser una enseñanza impartida por los propios profesionales, normalmente fuera de las universidades y con un cierto aire de religiosidad. HIERRO aboga por una enseñanza «laica» de la deontología de las profesiones jurídicas y que pueda ser integrada en los planes de estudio habituales de las facultades de Derecho; para ello tiene que redefinir en cierto sentido qué haya que entender por normas deontológicas, precisando de qué tipo de normas estamos hablando.

HIERRO ordena las distintas posibilidades en las que pueden encajar las normas deontológicas en la tradicional división entre normas jurídicas, normas morales y reglas de trato social. Pone de relieve que hay ejemplos de reconstrucción de todos estos tipos y algún caso de eclecticismo. Precisamente este último, sería el que HiERro entiende que refleja en mayor medida el uso que los cultivadores de la deontología hacen de la misma. Así, nos dirá que «por "deontología profesional" se entiende — siguiendo a LEGA- el conjunto de normas sociales, morales y jurídicas, de carácter no técnico, que regulan una profesión» (p. 283).

También sienta las bases para plantear una posible disciplina académica al respecto: «La deontología normativa requiere, por tanto: a) justificar el rol, b) justificar la organización del rol, y $c$ ) justificar los derechos y obligaciones excepcionales que se derivan del rol y de su organización» (p. 286). Resulta interesante el triple modo de justificación de las profesiones que el autor toma de Daryl KoEHN:

El primero sería el modelo del experto (expertise model): el profesional es un experto poseedor de ciertos conocimientos más o menos esotéricos que presta por licencia del Estado conforme a los estándares vigentes en su propia organización y dotado de gran autonomía; el protagonismo corresponde, en este primer modelo, al profesional. El segundo es el modelo contractual (contractualist model): el profesional es un prestador de servicios en el mercado; la relación del profesional con el cliente es una relación contractual y el protagonismo corresponde ahora al cliente. El tercero — que es el que propone KOEHN— es el modelo del compromiso público (public pledge), según el cual el profesional está vinculado por las expectativas del público respecto a las funciones que ha prometido desempeñar (p. 287).

${ }^{4}$ En L. HierRo, «Las profesiones jurídicas: una visión de conjunto», en Sistema, núm. 137, 1997, 27-44.

5 En L. HierRo, «Deontología de las profesiones jurídicas. Una discusión académica», Teoría y Derecho. Revista de pensamiento jurídico, núm. 8, 2010, 81-98. Publicado posteriormente en C. GARCía PASCUAL (coord.), El buen jurista. Deontología del Derecho, Valencia, Tirant lo Blanch, 2013, 269-301, por donde se cita. 


\section{MODELO DE PROFESIONAL Y ENSEÑANZA DEL DERECHO}

Creo que si uno tirara del hilo de este tercer modelo se abrirían expectativas de enseñanza académica muy interesantes. En algún sentido supondría darle a la enseñanza de la deontología profesional un mayor empaque académico, no simplemente por mor de privilegiar una especie de visión universitaria de las profesiones, sino por el reconocimiento de que el ejercicio de las mismas no puede llevarse a cabo desconociendo este compromiso público. Por eso, la conclusión a la que llega HiERro tal vez sepa a poco: «La deontología no establece ideales de vida, sino obligaciones y derechos propios de una actividad profesional. Solo establece qué es lo que los miembros de un determinado grupo profesional deben hacer y no deben hacer» (p. 301).

Habría, pues, que explorar de forma más detenida las implicaciones del tercer modelo citado, lo cual no tiene por qué llevar a la defensa de un perfeccionismo injustificado, que parece preocuparle al autor. Se trata simplemente de sacar las consecuencias pertinentes de un modelo de sociedad (que es liberal, en el sentido precisamente de no perfeccionista) respecto al rol de este tipo de profesiones.

Y aquí es donde se muestra pertinente retomar la clasificación de las profesiones jurídicas y su relación con la enseñanza del derecho en general. Se aprecia, por lo que acabamos de ver, que la delimitación de las funciones de las profesiones jurídicas debería marcar indefectiblemente la forma de enseñar la concreta materia de deontología jurídica, pero también debería permear la entera enseñanza del derecho. Si admitimos que este tercer modelo - de la profesión jurídica entendida como un cierto compromiso público- define mejor lo que esperamos hoy en día de un buen profesional del derecho, entonces parece que dos de los modelos de enseñanza del derecho de los que hablaba LAPORTA tienden a coincidir. En efecto, el modelo profesionalizador, una vez redefinido el rol de las profesiones jurídicas teniendo en cuenta su compromiso público, viene a solaparse con el modelo educador. Si esto es así, habría que resaltar (y más allá de una hipotética asignatura de deontología jurídica) la importancia del compromiso público que adquiere todo jurista, y justificaría, también desde el punto de vista del modelo profesionalizador, que se impartieran materias propias del modelo educador, así como la orientación de la «aplicación» (actividad que es definitoria de al menos algunas profesiones jurídicas, según HIERRO) del derecho mediatizada, entre otras cosas, por principios jurídicos con indudable carga moral. 